\title{
Measurement of protease activity of live Uronema marinum (Ciliata: Scuticociliatida) by fluorescence polarization
}

\author{
Eun Hye Lee, Chun Soo Kim, Jae Bum Cho, Kyoung Jin Ahn, Ki Hong Kim* \\ Department of Aquatic Life Medicine, Pukyong National University, Pusan 608-737, Korea
}

\begin{abstract}
The proteolytic activity of live Uronema marinum was analyzed by a fluorescence polarization (FP) technique. Protease activity was measured as a decrease in the FP value using fluorescein isothiocynate (FITC)-casein as a protein substrate. A time-dependent decrease in FP occurred in plate wells containing live $U$. marinum. Supplementation with the cysteine protease inhibitor E-64 had no significant inhibitory effect on the decrease in FP at any of the concentrations used. In contrast, supplementation with 1,10-phenanthroline resulted in complete inhibition of proteolysis for $30 \mathrm{~min}$ at $1 \mathrm{mM}$ and for $1 \mathrm{~h}$ at 2 and $5 \mathrm{mM}$. Effective inhibition of the proteolytic activity of live $U$. marinum by 1,10 -phenanthroline indicated that metalloproteases are the main proteases excreted by $U$. marinum. As $U$. marinum has a high potential for systemically invading and destroying fish tissues, the metalloproteases excreted by live $U$. marinum are likely to be involved in the invasion of host tissues and the pathogenicity of the parasite.
\end{abstract}

KEY WORDS: Uronema marinum · Proteases · Fluorescence polarization

Resale or republication not permitted without written consent of the publisher

Scuticociliatosis is defined as invasive external or systemic infection by protozoan ciliates belonging to the genera Uronema, Miamiensis and Philasterides, and is recognized as a fatal disease in marine fishes (Thompson \& Moewus 1964, Cheung et al. 1980, Yoshinaga \& Nakazoe 1993, Dyková \& Figueras 1994, Dragesco et al. 1995, Gill \& Callinan 1997, Munday et al. 1997, Sterud et al. 2000, Iglesias et al. 2001). Recent outbreaks of scuticociliatosis caused by $U$. marinum have resulted in severe mortality in the cultured olive flounder Paralichthys olivaceus in Korea (Jee et al. 2001).

In the pathogenesis of parasitic diseases, proteases have been shown to play important roles in host-tissue invasion, digestion of host proteins, and protection against immunological attacks by the host (McKerrow
1989). As Uronema marinum has high potential for systemically invading and destroying fish tissues (Cheung et al. 1980, Sterud et al. 2000, Jee et al. 2001), proteases excreted by the parasite may play a major role in the invasion of host tissues.

Although there have been many studies on proteases from several parasitic protozoan species (Mc Kerrow et al. 1993), little information is available on the proteolytic activities of parasitic scuticociliates. In the present study, the proteolytic activity of live Uronema marinum was analyzed by the fluorescence polarization technique.

Materials and methods. Culture, assays and analysis were carried out as follows:

Isolation and culture of Uronema marinum: $U$. marinum was isolated from the brain of infected olive flounder Paralichthys olivaceus and cultured in Eagle's minimum essential medium (MEM; Sigma Chemical) containing $10 \%$ fetal bovine serum (FBS; Sigma), $200 \mathrm{U} \mathrm{ml}^{-1}$ penicillin G (Sigma), and $200 \mathrm{U} \mathrm{ml}^{-1}$ streptomycin (Sigma) at $20^{\circ} \mathrm{C}$. Parasites harvested from the cultures were washed 3 times in phosphate-buffered saline (PBS) containing penicillin $\mathrm{G}$ and streptomycin by centrifugation at $1000 \times g$ for $5 \mathrm{~min}$ at $4^{\circ} \mathrm{C}$.

Protease assay: Instruments are now available to measure protease activity by fluorescence polarization (FP; Schade et al. 1996), a kinetic assay that measures in real time the hydrolysis in solution of a protein substrate previously conjugated with a fluorescent ligand. FP is based on the principle that a fluorescently labeled compound excited by plane-polarized light will emit polarized fluorescent light on the same plane if there is minimal molecular movement between the excitation and emission times. The smaller the molecular mass of the labeled molecule, the greater the movement will be, and the lower the emitted fluorescence polarization. Cleavage of large fluorescently labeled substrate 
molecules by addition of a protease results in a protease concentration-dependent decrease in FP as the more mobile protease digestion products are released and rapidly rotate to emit fluorescence from the excitation plane of polarization. Therefore, the measure of the decrease in fluorescence polarization is the measure of proteolytic activity.

Proteolytic activity of Uronema marinum was determined by incubating $5 \times 10^{4}$ cells of the parasite in $190 \mu \mathrm{l}$ PBS (pH 7.0) with $10 \mu \mathrm{l}$ of $10 \mu \mathrm{g} \mathrm{ml}^{-1}$ fluorescein

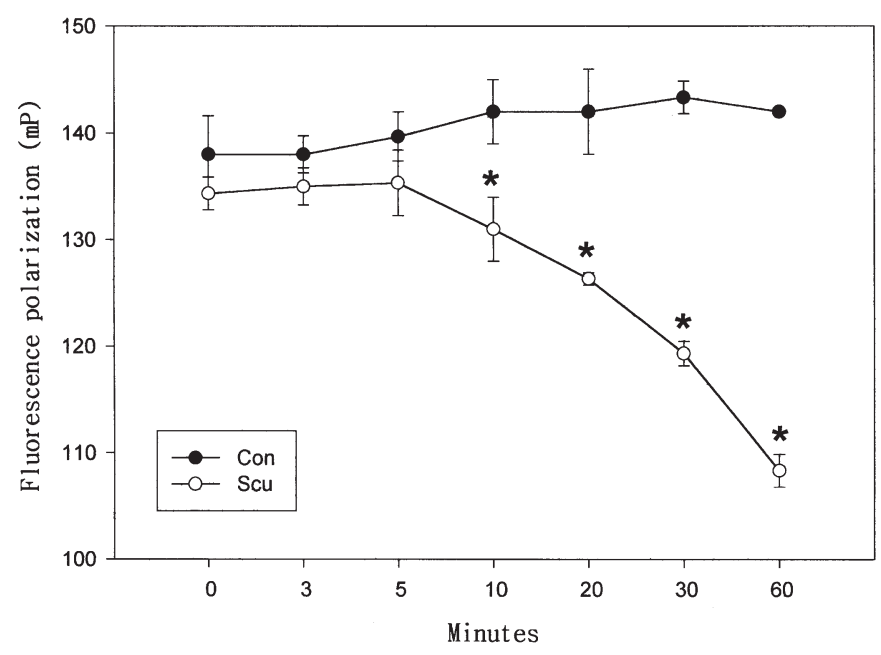

Fig. 1. Uronema marinum. Protease activity (millipolorization units, means $\pm \mathrm{SD}$ ) of live parasites (Scu) measured by fluorescence polarization. Proteolytic activity was detected using FITC-casein as a substrate at $\mathrm{pH} 7 .{ }^{*}$ Significantly $(\mathrm{p}<0.05)$ lower than control (Con)

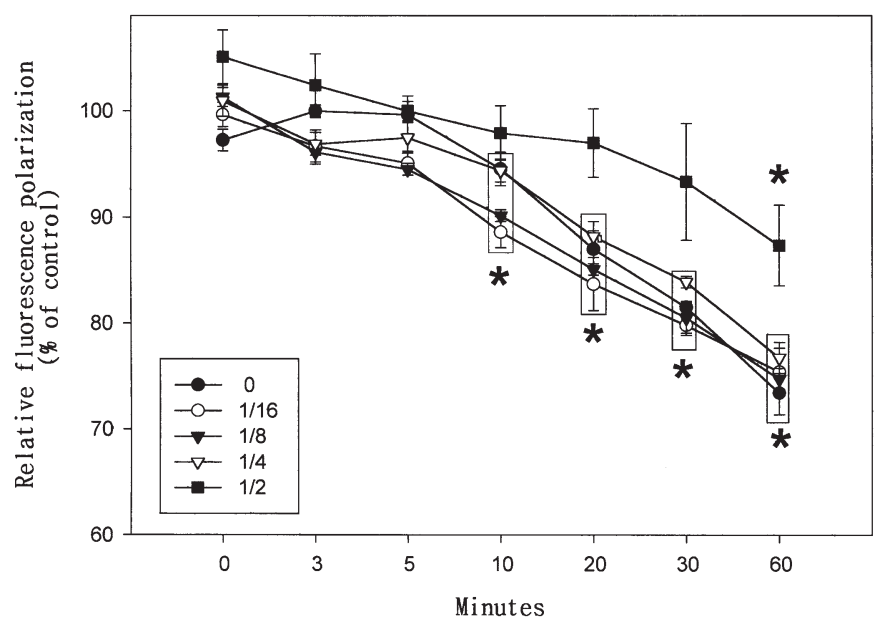

Fig. 2. Uronema marinum. Inhibition of (means $\pm \mathrm{SD}$ ) protease activity of live parasites by various dilutions of protease inhibitor cocktail. Protease activity was measured by fluorescence polarization, and was detected using FITC-casein as a substrate at $\mathrm{pH} 7 .{ }^{*}$ Significantly $(\mathrm{p}<0.05)$ lower than relative controls isothiocynate (FITC)-casein (Sigma) in a black 96-well plate (Greiner Bio-One) at $25^{\circ} \mathrm{C}$. Wells without live parasites were used as controls and wells with only PBS were designated blanks. Fluorescence polarization was measured with a Polarion instrument (TECAN). The excitation wavelength was $485 \mathrm{~nm}$ and the emission wavelength $535 \mathrm{~nm}$. All assays were done in triplicate. Readings were automatically recorded at $0,3,5,10,30$ and $60 \mathrm{~min}$ in millipolarization units (mP).

Polarization $(P)$ is defined in Eq. (1), where $I_{V V}$ is the fluorescence intensity when both the excitation and emission polarizers are parallel, and $I_{v h}$ is the fluorescence intensity when the excitation and emission polarizers are perpendicular:

$$
P=\left(I_{V V}-G \times I_{V h}\right) /\left(I_{V V}+G \times I_{V h}\right)
$$

The instrument factor, $G$, was set such that $1 \mathrm{nM}$ of FITC solution yielded a polarization value of $20 \mathrm{mP}$.

Effects of protease inhibitors: Using FITC-casein as a substrate, the effects of protease inhibitor cocktail (Sigma), trans-epoxysuccinyl-L-leucylamido-(4-guanidino) butane (E-64, Sigma), and 1,10-phenanthroline (Sigma) on the proteolytic activity of live Uronema marinum were examined. The protease inhibitor cocktail contained 4-(2 aminoethyl)benzenesulfonyl fluoride, E-64, bestatin, leupeptin, aprotinin, and sodium EDTA. Each inhibitor was diluted with PBS (pH 7.0), and the final concentrations of each inhibitor in $200 \mu \mathrm{l}$ of a total reaction volume were $1 / 2,1 / 4,1 / 8$, and $1 / 16$ dilutions of the protease inhibitor cocktail, 50, 100 and $200 \mu \mathrm{M}$ of E-64, and 1, 2 and $5 \mathrm{mM}$ of 1,10-phenanthroline. The other experimental conditions were similar to those described in the protease assay. Wells with FITC-casein and each concentration of each inhibitor without the parasite were used as controls, and wells containing only $200 \mu \mathrm{l}$ PBS were designated blanks. The level of inhibition was expressed as a percentage of the FP value relative to each control FP value. All assays were done in triplicate.

Statistical analysis: A Student's $t$-test was used to determine the statistical difference between each concentration of inhibitors and each relative control. Results were considered significant if $\mathrm{p}<0.05$.

Results. A time-dependent decrease in FP occurred in the plate wells containing live Uronema marinum (Fig. 1). In contrast, control wells containing only FITCcasein showed no decrease in FP. The effect of the protease inhibitor cocktail on the FP assay is shown in Fig. 2. The wells supplemented with $1 / 16,1 / 8$ and $1 / 4$ dilutions of the protease inhibitor cocktail showed a significant decrease in FP after 10 min incubation. However, the wells supplemented with $1 / 2$ dilution of the cocktail showed no significant decrease in FP until after 30 min incubation. Supplementation with E-64, a cysteine protease inhibitor, had no significant inhibitory effect 
on the decrease in FP at any of the inhibitor concentrations used (Fig. 3). In contrast, supplementation with 1,10-phenanthroline, a metalloprotease inhibitor, resulted in complete inhibition of proteolysis for $30 \mathrm{~min}$ at $1 \mathrm{mM}$ and for $1 \mathrm{~h}$ at 2 and $5 \mathrm{mM}$ (Fig. 4).

Discussion. In the present study, proteolytic activity of live Uronema marinum was demonstrated by FP. The gradual drop in FP values with increasing incubation time indicates that the FITC-casein was cleaved by increasing concentrations of proteases excreted by the parasites. Although Schade et al. (1996) used the

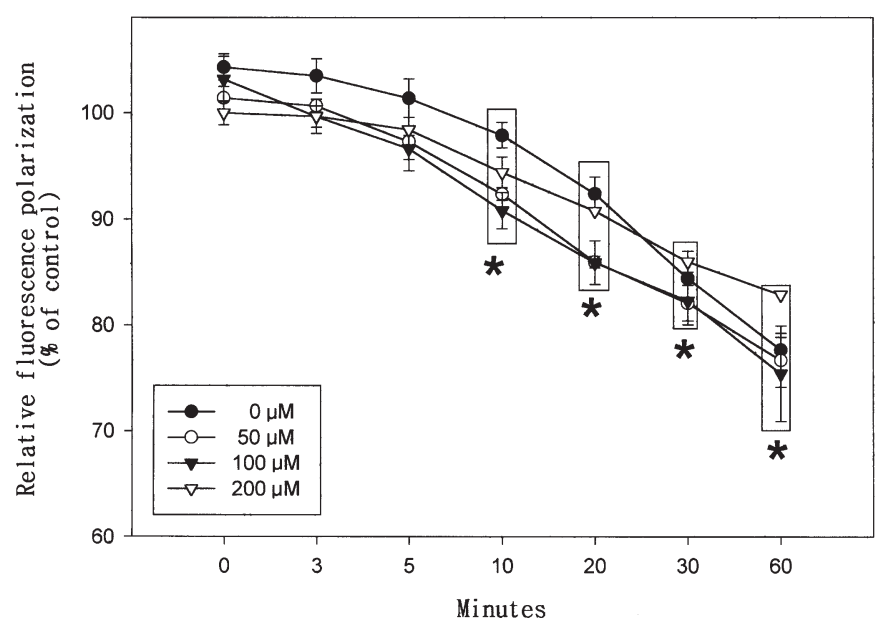

Fig. 3. Uronema marinum. Inhibition (means \pm SD) of protease activity by cysteine protease inhibitor E-64. Protease activity was measured by fluorescence polarization, and was detected using FITC-casein as a substrate at $\mathrm{pH}$ 7. * : Significantly ( $\mathrm{p}<$ $0.05)$ lower than relative controls

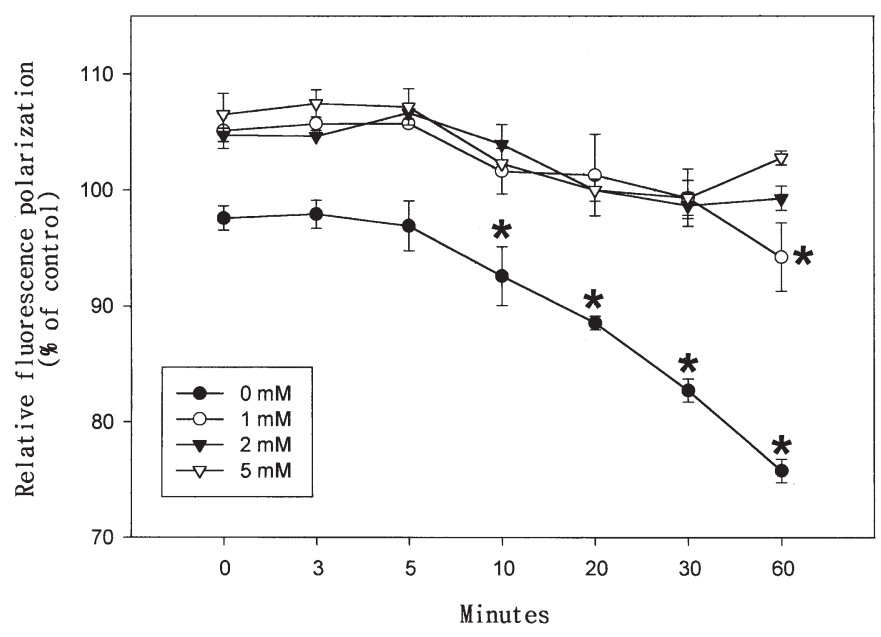

Fig. 4. Uronema marinum. Inhibition of protease activity by metalloprotease inhibitor 1,10-phenanthroline. Protease activity was measured by fluorescence polarization, and was detected using FITC-casein as a substrate at pH 7. * : Significantly $(p<0.05)$ lower than relative controls for 1,2 and $5 \mathrm{mM}$ concentrations
FP assay to measure proteolytic activity of whole bacteria (Porphyromonas gingivalis), as far as we know there are no previous reports on the measurement of proteolytic activity of live protozoans using the FP assay. In comparison to other assays of protease activity, including radioactive counting, spectrophotometric or spectrofluorometric analysis and gel electrophoresis, the FP assay has the advantage of providing measurements of substrate hydrolysis by live parasites in real time. Because measurements are taken in real time, the progress of the reaction is followed both kinetically and at single points in time. Additionally, the FP assay is homogeneous and, as such, does not require separation, precipitation, or transfer of the reaction mixture (Bolger \& Checovich 1994).

Cysteine proteases in parasitic protozoans have been assumed to have metabolic and physiological roles (North 1992). In Trypanosoma cruzi, the function of cystein protease is to degrade intracellular protein and remodel the parasite during transformation between stages (Harth et al. 1993). Cysteine proteases are critical to host invasion for a number of human protozoan parasites including T. cruzi (Franke de Cazzulo et al. 1994), Plasmodium falciparum (Mayer et al. 1991), Cryptosporidium parvum (Forney et al. 1996), and Entamoeba histolytica (Que \& Reed 2000). In the present study, no significant inhibition of proteolytic activity of the parasite by E-64 at concentrations of up to $200 \mu \mathrm{M}$ at physiological $\mathrm{pH}$ occurred, indicating that cysteine proteases may not be involved in this parasite's invasion of fish tissues.

In the present study, effective inhibition of the proteolytic activity of live Uronema marinum by the metalloprotease inhibitor 1,10-phenanthroline indicated that metalloproteases are the main proteases excreted by $U$. marinum. As $U$. marinum has a high potential for systemically invading and destroying fish tissues, the metalloproteases excreted by the parasite could be involved in the invasion of host tissues and the pathogenicity of the parasite. Metalloproteases in some parasitic protozoans contribute to invasion and degradation of host tissues and thus play an important role in the progress of the disease (McKerrow et al. 1993). The functions of metalloproteases in pathogenesis have been well documented for Cryptobia salmositica, a haemoflagellate parasite of fishes (Zuo \& Woo 1997a,b, 1998a,b, 2000). Further research into the biochemical nature and the functions of the metalloproteases secreted by $U$. marinum may provide us with a better understanding of the disease process in scuticociliatosis.

Acknowledgements. This work was supported by grant no. R01-2000-000-00215-0 of the Basic Research Program of the Korea Science \& Engineering Foundation. 


\section{LITERATURE CITED}

Bolger R, Checovich W (1994) A new protease activity assay using fluorescence polarization. Biotechniques 17: 585-589

Cheung PJ, Nigrelli RF, Ruggieri GD (1980) Studies on the morphology of Uronema marinum Dujardin (Ciliatea: Uronematidae) with a description of the histopatology of the infection in marine fishes. J Fish Dis 3:295-303

Dragesco A, Dragesco J, Coste F, Gasc C, Romestand B, Raymond J, Bouix G (1995) Philasterides dicentrarchi, n. sp. (Ciliophora, Scuticociliatida), a histophagous opportunistic parasite of Dicentrachus labrax (Linnaeus, 1758), a reared marine fish. Eur J Protistol 31:327-340

Dyková I, Figueras A (1994) Histopathological changes in turbot Scophthalmus maximus due to a histophagous ciliate. Dis Aquat Org 18:5-9

Forney JR, Yang S, Healey MC (1996) Protease activity associated with excystation of Cryptosporidium parvum oocysts. J Parasitol 82:889-892

Franke de Cazzulo BM, Martinez J, North M, Coombs GH, Cazzulo JJ (1994) Effects of proteinase inhibitors on the growth and differentiation of Trypanosoma cruzi. FEMS Microbiol Lett 124:81-86

Gill PA, Calinan RB (1997) Ulcerative dermatitis associated with Uronema sp. infection of farmed sand whiting Sillago ciliata. Aust Vet J 75:357

Harth G, Andrews N, Mills AA, Engel JC, Smith R, McKerrow JH (1993) Peptide-fluoromethyl ketones arrest intracellular replication and intercellular transmission of Trypanosoma cruzi. Mol Biochem Parasitol 58:17-24

Iglesias R, Paramá A, Alvarez MF, Leiro J, Fernández J, Sanmartín ML (2001) Philasterides dicentrarchi (Ciliophora, Scuticociliatida) as the causative agent of scuticociliatosis in farmed turbot Scophthalmus maximus in Galicia (NW Spain). Dis Aquat Org 46:47-55

Jee BY, Kim YC, Park MS (2001) Morphology and biology of parasite responsible for scuticociliatosis of cultured olive flounder Paralichthys olivaceus. Dis Aquat Org 47:49-55

Mayer R, Picard I, Lawton P, Grellier P, Barrault C, Monsigny M, Schrevel J (1991) Peptide derivatives specific for a Plasmodium falciparum proteinase inhibit the human erythrocyte invasion by merozoites. J Med Chem 34: 3029-3035

Editorial responsibility: Wolfgang Körting,

Hannover, Germany
McKerrow JH (1989) Minireview: parasite proteases. Exp Parasitol 68:111-115

McKerrow JH, Sun E, Rosenthal PJ, Bouvier J (1993) The proteases and pathogenicity of parasitic protozoa. Annu Rev Microbiol 47:821-853

Munday BL, O'Donoghue PJ, Watts M, Rough K, Hawkesford $\mathrm{T}$ (1997) Fatal encephalitis due to the scuticociliate Uronema nigricans in sea-caged, southern bluefin tuna Thunnus maccoyii. Dis Aquat Org 30:17-25

North MJ (1992) The characteristics of cysteine proteases of parasitic protozoa. Biol Chem 373:401-406

Que X, Reed SL (2000) Cysteine proteinases and the pathogenesis of amebiasis. Clin Microbiol Rev 13:196-206

Schade SZ, Jolley ME, Sarauer BJ, Simonson LG (1996) BODIPY- $\alpha$-casein, a $\mathrm{pH}$-independent protein substrate for protease assays using fluorescence polarization. Anal Biochem 243:1-7

Sterud E, Hansen MK, Mo TA (2000) Systemic infection with Uronema-like ciliates in farmed turbot, Scophthalmus maximus (L.). J Fish Dis 23:33-37

Thompson CL Jr, Moewus L (1964) Miamiensis avidus n. g. n. s., a marine facultative parasite in the ciliate order Hymenostomatida. J Protozool 11:378-381

Yoshinaga T, Nakazoe J (1993) Isolation and in vitro cultivation of an unidentified ciliate causing scuticociliatosis in Japanese flounder (Paralichthys olivaceus). Fish Pathol 28:131-134

Zuo X, Woo PTK (1997a) Proteases in pathogenic and nonpathogenic haemoflagellates, Cryptobia spp. (Sarcomastigophora: Kinetoplastida), of fishes. Dis Aquat Org 29:57-65

Zuo X, Woo PTK (1997b) Purified metallo-protease from the pathogenic haemoflagellate Cryptobia salmositica and its in vitro proteolytic activities. Dis Aquat Org 30:177-185

Zuo X, Woo PTK (1998a) Characterization of purified metalloand cysteine proteases from the pathogenic haemoflagellate, Cryptobia salmositica Katz, 1951. Parasitol Res 84: 492-498

Zuo X, Woo PTK (1998b) In vitro secretion of the metalloprotease by the pathogenic haemoflagellate Cryptobia salmositica Katz, 1951 and stimulation of the protease production by collagen. J Fish Dis 21:249-255

Zuo X, Woo PTK (2000) In vitro haemolysis of piscine erythrocytes by purified metallo-protease from the pathogenic haemoflagellate, Cryptobia salmositica Katz. J Fish Dis 23:227-230

Submitted: September 4, 2002; Accepted: December 11, 2002 Proofs received from author(s): February 25, 2003 